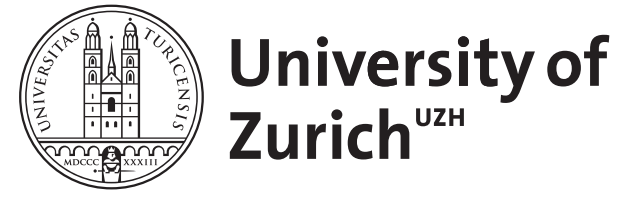

\title{
Developments in targeted therapy in melanoma
}

\author{
Amann, V C ; Ramelyte, E ; Thurneysen, S ; Pitocco, R ; Bentele-Jaberg, N ; Goldinger, S M ;
}

Dummer, R ; Mangana, J

\begin{abstract}
Melanomas are disease entities driven in part by the mitogen activated protein kinase (MAPK) pathway. The TCGA network recently defined four genetic subtypes based on the most prevalent significantly mutated genes, including mutant BRAF, mutant RAS (N/H/K), mutant NF1, and Triple wild-type melanoma (harboring none of the aforementioned mutations, but instead includes KIT, GNA and GNAQ mutations). The successful development of kinase inhibitors marked a milestone in the treatment of metastatic melanoma. Combination treatment with a BRAF- and MEK-inhibitor is the current standard of care for inoperable stage IIIC/IV BRAF-mutated melanoma. Recent data demonstrate excellent longterm outcome, especially in patients with normal baseline LDH levels, and confirm that there is a subset of BRAF inhibitor-naive patients who experience durable responses without progression on combination treatment. In the future, adding a third compound based on individual genetic alterations might further improve the outcome of targeted therapy.
\end{abstract}

DOI: https://doi.org/10.1016/j.ejso.2016.10.014

Posted at the Zurich Open Repository and Archive, University of Zurich ZORA URL: https://doi.org/10.5167/uzh-128596

Journal Article

Accepted Version

Originally published at:

Amann, V C; Ramelyte, E; Thurneysen, S; Pitocco, R; Bentele-Jaberg, N; Goldinger, S M; Dummer, R; Mangana, J (2017). Developments in targeted therapy in melanoma. European Journal of Surgical Oncology, 43(3):581-593.

DOI: https://doi.org/10.1016/j.ejso.2016.10.014 


\title{
Review
}

\section{Developments in targeted therapy in melanoma}

\author{
V.C. Amann ${ }^{\mathrm{a}, \mathrm{b}}$, E. Ramelyte ${ }^{\mathrm{a}, \mathrm{c}}$, S. Thurneysen ${ }^{\mathrm{a}}$, R. Pitocco ${ }^{\mathrm{d}}$, \\ N. Bentele-Jaberg ${ }^{a}$, S.M. Goldinger ${ }^{\text {a }}$, R. Dummer ${ }^{\text {a, } *}$, J. Mangana ${ }^{a}$ \\ ${ }^{a}$ Department of Dermatology, University Hospital Zurich, Zurich, Switzerland \\ ${ }^{\mathrm{b}}$ University Department of Medicine, Kantonsspital Aarau, Tellstrasse, 5001 Aarau, Switzerland \\ ${ }^{\mathrm{c}}$ Vilnius University, Centre of Dermatovenereology, Vilnius, Lithuania \\ ${ }^{\mathrm{d}}$ Department of Dermatology, University of Rome Tor Vergata, Rome, Italy
}

Accepted 24 October 2016

Available online

\begin{abstract}
Melanomas are disease entities driven in part by the mitogen activated protein kinase (MAPK) pathway. The TCGA network recently defined four genetic subtypes based on the most prevalent significantly mutated genes, including mutant $B R A F$, mutant $R A S$ (N/H/K), mutant NF1, and Triple wild-type melanoma (harboring none of the aforementioned mutations, but instead includes KIT, GNA and GNAQ mutations).

The successful development of kinase inhibitors marked a milestone in the treatment of metastatic melanoma. Combination treatment with a BRAF- and MEK-inhibitor is the current standard of care for inoperable stage IIIC/IV BRAF-mutated melanoma. Recent data demonstrate excellent long-term outcome, especially in patients with normal baseline LDH levels, and confirm that there is a subset of BRAF inhibitor-naive patients who experience durable responses without progression on combination treatment. In the future, adding a third compound based on individual genetic alterations might further improve the outcome of targeted therapy.

(C) 2016 Elsevier Ltd, BASO The Association for Cancer Surgery, and the European Society of Surgical Oncology. All rights reserved.
\end{abstract}

\section{Introduction}

In the past decade, revolutionary insights were made in understanding and treating melanoma. The identification of the critical role of the mitogen activated protein kinase (MAPK) pathway and development of targeted therapy dramatically changed prognosis and overall survival (OS) of metastatic melanoma patients. ${ }^{1,2}$ Novel techniques such as next generation sequencing enable the identification of new cancer "driver" genes and their mutations. These new insights contribute to prognostication and create opportunities for development of innovative mutationdirected therapies.

\footnotetext{
* Corresponding author. University Hospital of Zurich, Department of Dermatology, Gloriastrasse 31, CH-8091 Zürich, Switzerland. Fax: +41 442558988 .

E-mail address: reinhard.dummer@usz.ch (R. Dummer).
}

Sequencing data have shown that the median mutation rate in melanoma is $>10$ mutations $/ \mathrm{Mb}$, the highest of all cancers so far analyzed by The Cancer Genome Atlas (TCGA) network. ${ }^{3}$ Nevertheless, the number of mutations differs according to the site, with the lowest rate in primary melanomas on non-ultraviolet-exposed non-glabrous skin and the highest in patients with history of chronic sun exposure. ${ }^{4}$ Genetic alterations in melanoma oncogenes and tumor suppressor genes commonly cause constitutive signaling through RAS-RAF-MEK-ERK, also known as the MAPK pathway. ${ }^{5,6}$ This cascade concludes in activation of ERK1 and ERK2, which can then translocate to the nucleus and regulate MITF, c-MYC and other transcription factors, resulting in alteration of cell proliferation and senescence (Fig. 1). ${ }^{6}$ Less frequently identified, yet also relevant are genetic aberrations in other cellular pathways, such as cell cycle control (CDKN2A), apoptosis (PT53) and the PI3K pathway $(T E R T, P T E N) .^{7-10}$ In 2015, TCGA 


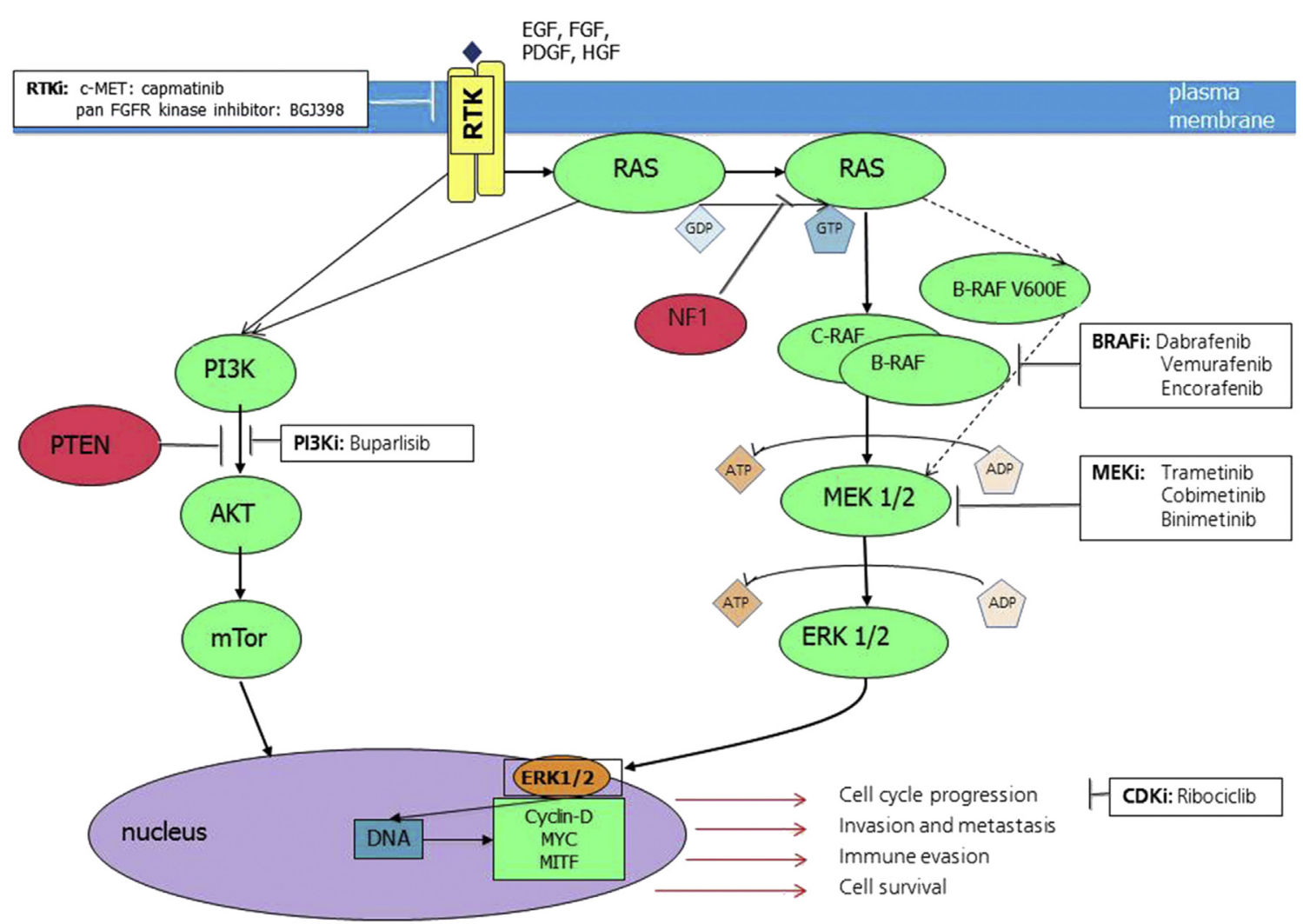

Figure 1. (Oncogenes shown in green, tumor suppressors shown in red) Extracellular signals such as EGF (epidermal growth factor), FGF (fibroblast growth factor), PDGF (platelet derived growth factor) or HGF (hepatocyte growth factor) bind to corresponding receptors (EGFR, FGFR, PDGFR and c-Myc, respectively) and induce signal transduction, which results in activation of RAS (exchange of protein-bound GDP (inactive form) to GTP (active form), accelerated by guanine nucleotide exchange factors). ${ }^{13}$ NF1 negatively regulates RAS by increasing RAS GTPase activity and hence turning RAS-GTP to RAS-GDP. ${ }^{8}$ Active RAS leads to activation of BRAF (dimerization of non-mutated BRAF; BRAF V600E mutated protein can be active as a monomer) and subsequently MEK. This cascade concludes in activation of ERK1 and ERK2, which can then translocate to the nucleus and regulate MITF, c-MYC and other transcription factors, resulting in alteration of cell proliferation and senescence. ${ }^{6}$ Receptor tyrosine kinases (RTK) or RAS-GTP can activate the PI3K-AKT pathway, while PTEN is a negative regulator, mutation of which leads to constitutive activation of AKT and is followed by changes in cell growth, motility and invasion. ${ }^{124}$

Network suggested a new genomic classification for melanoma based on the most prevalent significantly mutated genes: mutant $B R A F$, mutant $R A S$, mutant $N F 1$ and triple wild-type (triple $w t$ ) melanoma. ${ }^{7}$

\section{Genetic landscape of mutations}

The most common genetic alteration, accounting for around $50 \%$ of all somatic mutations in cutaneous melanoma, is activating mutation of serine-threonine kinase $B R A F$ gene. ${ }^{7,8,11}$ This causes constitutive activation of BRAF protein, which results in increased proliferation and survival of melanoma cells. ${ }^{12}$ In more than $90 \%$ of cases valine is substituted with glutamate at codon 600 (V600E), less frequently with lysine (V600K) or arginine (V600R) ${ }^{5,7}$ Interestingly, patients with $B R A F$ mutations are generally younger. It is most frequent in cutaneous melanoma, while it's detected in only $10-20 \%$ of mucosal melanoma. ${ }^{5}$

RAS is a member of the guanosine $5^{\prime}$-triphosphatase (GTP)-binding protein family. Under normal conditions, RAS activation is induced by extracellular signaling and is reversible. It's driven by guanine nucleotide exchange factor, which results in exchange of protein-bound guanosine diphosphate (GDP) to triphosphate (GTP). Mutations in the $R A S$ gene impair inactivation and keep RAS protein in the activated state. ${ }^{13}$ The active form, RAS-GTP, initiates phosphorylation of RAF and subsequently MEK and ERK and leads to the activation of the MAPK pathway. ${ }^{13}$ This specific structure causes difficulties in targeting; hence, RAS was named the "undruggable" target. ${ }^{13}$ RAS mutations are found in around 30\% of melanomas and usually affect NRAS (Q61R, Q61K, and Q61H). ${ }^{7,11,14}$ RAS not only activates the MAPK pathway, but also has activators and effectors among other cellular pathways such as phosphatidylinositol-3 kinases (PI3K), T-Cell Lymphoma Invasion and Metastasis 1 (TIAM1) and others. ${ }^{13}$

The third most frequently identified genetic mutation is located in the NFI tumor suppressor gene, which serves as a regulator of RAS through GTP-ase activating protein. Due to an inactivating mutation, the regulative properties of NF1 are lost, which results in continuous activation of RAS. ${ }^{15}$ This mutation was observed in $14 \%$ of samples analyzed by $\mathrm{TCGA}^{7}$ and in $46.4 \%$ of BRAF and NRAS 
$w t$ melanomas analyzed by Krauthammer et al. ${ }^{8}$ Interestingly, around $60 \%$ of these melanomas harbored comutations in RAS-opathy genes (RASA2, PTPN11, SOS1, $R A F 1$ and SPREDI), ${ }^{8,16}$ which are known to be linked with Noonan, Leopard and Legius syndromes. ${ }^{8}$

Melanomas referred to as triple $w t$ show none of the three previously mentioned mutations. This subgroup includes $G N A Q$, commonly found in uveal melanoma, or KIT mutations. Interestingly, only $30 \%$ of triple $w t$ melanomas harbor a UV signature, compared to over $90 \%$ of $B R A F$-, RAS- and NFl-subtypes, but more copy number changes and complex structural arrangements are identified in triple $w t^{7}$

Identifying mutations open opportunities for the development of new therapeutic agents, identification of predictive biomarkers and aid in understanding of melanoma genesis. Shain et al. evaluated genetic alterations in 37 primary melanomas and their adjacent antecedent lesions. ${ }^{17}$ Interestingly, histologically benign lesions harbored V600E mutations only, while intermediate lesions harbored additional $B R A F$ as well as NRAS mutations. Compared to benign and intermediate lesions, more Telomerase reverse transcriptase (TERT) promoter and copy-number alterations were identified in melanoma, whereas Phosphatase and tensin homolog (PTEN) and tumor protein p53 (TP53) mutations were only found in advanced primary melanomas. ${ }^{7,17}$ This suggests that $B R A F$ mutations are sufficient for nevus formation, but additional oncogenic alterations are needed for malignant transformation.

\section{Driver mutations as prognostic factors in melanoma?}

One of the major questions raised in the last years is whether the identification of driver mutations has prognostic or predictive clinical significance.

Long et al. were initially able to demonstrate an association between BRAF mutations and inferior clinical outcome in a prospective cohort of 197 Australian melanoma patients, albeit with no influence on disease free survival (DFS) ${ }^{18}$ Similarly, NRAS mutant melanoma patients were also reported to have impaired survival with a higher incidence of central nervous system (CNS) involvement in a retrospective setting; however no difference in overall survival (OS) between $B R A F$ mutated and $w t$ melanomas was noted. ${ }^{19}$

Since then, several studies have been conducted, none of which were able to show an influence of BRAF or NRAS mutation status on OS. ${ }^{20-26}$

A subsequent prospective Australian study $(\mathrm{n}=308)$ was not able to confirm the previously reported impact on OS of BRAF mutant melanoma patients. However, oneyear survival from diagnosis of metastasis was significantly longer for patients with $B R A F$ mutant melanoma treated with a kinase inhibitor than those without $(29 \%$, $\mathrm{p}<0.001)$, or for BRAF wt patients $(37 \%, \mathrm{p}<0.001) .{ }^{27}$ Similarly, Frauchiger et al. confirmed that $B R A F$ mutant melanoma patients treated with selective kinase inhibitors have a statistically significant longer OS compared to $w t$ patients (OS 14.5 vs 10.6 months, $\mathrm{p}=0.14$ ). ${ }^{24}$

Relating to immunotherapy, it has recently been shown that harboring an NRAS mutation increases the response rate (RR) to checkpoint inhibitors with no significant impact on OS and progression-free survival (PFS). ${ }^{28,29} \mathrm{~A}$ recent retrospective study affirmed this assumption: although mutational status had no influence on OS in anti-CTLA-4 treated patients, a non-statisticallysignificant trend for superior clinical outcome in NRAS mutant patients was observed. ${ }^{30}$

As outlined previously, the third most commonly reported mutation is the inactivating mutation of NF1, which shows similar OS compared to BRAF-mutant, NRASmutant or triple $w t$ melanomas. ${ }^{8}$ No correlation between the loss of $N F 1$ and response to treatment with MEK or ERK inhibitors has been noted in vitro so far. ${ }^{8}$ Nevertheless, further large prospective clinical trials are needed to address this issue.

Besides these most commonly observed mutations in melanoma, other features seem to have prognostic impact. TERT promoter mutations are associated with impaired OS in cutaneous compared to acral, mucosal and uveal melanomas $^{31}$ and are present in approximately $70-80 \%$ of $B R A F-, N R A S$ - and NFI- compared to $7 \%$ of triple $w t$ melanomas. ${ }^{7}$ Furthermore, they were found in $61 \%$ of fastgrowing compared to $32 \%$ of slow-growing melanomas $(\mathrm{p}=<0.0001) .{ }^{32}$ Thus, it was suggested that the presence of TERT promoter mutations can partly explain a more aggressive clinical outcome, associated with an accelerated growth rate. In contrast, $B R A F$ and $N R A S$ hotspot mutations have not been found to be associated with increased growth. Therefore, TERT promoter mutation may serve as a future biomarker to identify aggressive tumors, which could benefit from adjuvant treatment.

The prognostic significance of PTEN promoter methylation in clinical outcome of melanoma patients was recently elucidated. ${ }^{9,33}$ In a cohort of 392 melanoma patients, PTEN was identified as an independent predictor for impaired survival, highlighting potential therapeutic opportunities in this field. ${ }^{9}$

In summary, the mutation status alone does not seem to be sufficient to predict the outcome of advanced melanoma patients and cannot be used as an independent prognostic factor. However, the identification of other genetic features and their eventual prognostic significance are fundamental steps in the field of melanoma management.

\section{Multi-kinase inhibitors}

\section{Tyrosine kinase inhibitors}

Among targeted therapies, monoclonal antibodies (mAbs) and small-molecule inhibitors (SMIs) of multiple tyrosine kinase activity present as ideal candidates for 
melanoma treatment. ${ }^{34}$ Tyrosine kinase inhibitors (TKIs) block vital pathways in the context of cell growth and survival via binding to small intercellular molecules, which are common phosphorylation sites of kinases (most commonly tyrosine or serine-threonine kinases) that are either associated with growth factor receptors or downstream signaling molecules. Several clinical trials have been performed over the last years either with single agents or in combination with chemotherapy. However, the demonstrated clinical benefit with multi-targeted tyrosine kinase inhibitors (mTKIs) was limited compared to selective BRAF and MEK inhibitors.

\section{Sorafenib}

Sorafenib is an orally available, broad-spectrum mTKI with anti-proliferative and anti-angiogenic effects. ${ }^{35}$ Sorafenib targets several RAF isoforms as well as other receptor tyrosine kinase inhibitors (RTKI) like vascular endothelial growth factor (VEGFR 2/3), platelet-derived growth factor receptor beta (PDGFR- $\beta$ ), fms-related tyrosine kinase 3 (FLT-3) and c-KIT. ${ }^{30,35}$

In clinical trials with advanced melanoma patients, sorafenib showed no clinical benefit as a single agent. ${ }^{36,37}$ In the context of evaluating combination therapies, Flaherty et al. presented encouraging preliminary results of sorafenib combined with carboplatin and paclitaxel (CP). ${ }^{38}$ The combination showed a $37 \%$ partial response (PR) rate in the interim analysis while another $48 \%$ demonstrated stable disease (SD) ${ }^{38}$ Nevertheless, phase III clinical trials failed to show significant benefit. ${ }^{39,40}$

In addition, several phase I/II studies with sorafenib in combination with dacarbazine (DTIC) or temozolomide (TMZ) showed low anti-tumor activity and mainly achieved SD. ${ }^{41-43}$ The beneficial $45.5 \%$ overall metabolic RR (PERCIST criteria) in a recent clinical trial did not result in lasting objective responses. ${ }^{44}$ Sorafenib in combination with $\mathrm{CP}$ in uveal melanoma patients also failed to achieve clinical benefit with the study being terminated early (PFS 4 months, OS 11 months, no confirmed objective tumor responses by RECIST criteria). ${ }^{45}$ However, the results of the STREAM study in chemo-naive metastatic uveal melanoma patients are still pending. ${ }^{46}$

In general, sorafenib monotherapy and combination therapies had a manageable toxicity profile, with the majority of adverse events (AEs) being mild. ${ }^{36,37,39-41,47}$ Severe adverse events (SAE) were reported in $51 \%$ of patients, with the most common being hematologic toxicities. ${ }^{47}$ The most commonly observed drug-related AEs under sorafenib monotherapy were skin reactions (rash and palmarplantar erythrodysesthesia syndrome), gastrointestinal and constitutional disorders (corresponding grade 1-2 intensity). ${ }^{36,37}$ Combination treatments with sorafenib led to hematotoxicity, fatigue, sensory neuropathy and skin reactions. ${ }^{39,40,43}$

Based on the limited activity of sorafenib, doubts were raised concerning the potential of RAF inhibition as a therapeutic option. Therapeutic failure of sorafenib in advanced melanoma was likely due to an inability to selectively achieve RAF inhibition at maximum tolerated doses. Subsequent trials with selective BRAF inhibitors (BRAFi) followed, changing the era of melanoma treatment.

\section{Pazopanib}

Pazopanib is another orally-bioavailable, adenosine triphosphate (ATP)-competitive TKI with selectivity for VEGFR-1, -2, and -3, FDA-approved for renal carcinoma and soft tissue sarcoma in $2009 .{ }^{48}$ Furthermore, it blocks the PDGFR- $\alpha$ and $-\beta$ as well as c-KIT.

Clinical trials in melanoma patients with pazopanib are limited. None of the studies published so far reported significant clinical benefit in advanced $B R A F$ wt melanoma patients. ${ }^{49,50}$ A recent single-center pilot study investigating the metabolic response, the early cytokine and chemokine profile and the histological findings of metastatic tissue under pazopanib and paclitaxel in the second line setting showed moderate efficacy. ${ }^{51} 17$ patients with stage III or IV melanoma were included. 5 out of 14 evaluable patients showed partial metabolic response (using PRECIST 1.0 criteria) after 10 days of pazopanib monotherapy. No response was achieved at day 70 of combination treatment. The median progression-free survival (mPFS) was 70 days, the median overall survival (mOS) 208 days. ${ }^{51}$

The drug was generally well tolerated with $87 \%$ of all AEs being mild to moderate, such as loss of appetite, weakness, skin reactions, bone marrow function impairment and neurological symptoms. $13 \%$ of AEs were grade 3 or grade 4 .

\section{Other multi-kinase inhibitors}

Axitinib, another tyrosine kinase inhibitor against VEGFR-1, -2, -3, PDGFR- $\beta$ and $c-K I T$, showed promising activity in combination with carboplatin and paclitaxel in $w t$ metastatic melanoma patients in a phase II clinical study (RR 18.8\%, 6-month-PFS of 33.9\%). ${ }^{52}$ However, these result warrants further testing in randomized phase III trials. On the other hand, the multi-kinase inhibitor Lenvatinib (E7080) achieved limited responses both in monotherapy and in combination with TMZ in the phase I setting. ${ }^{53}$

\section{KIT inhibitors}

The KIT receptor protein tyrosine kinase is a transmembrane protein consisting of extra- and intracellular binding domains and a signal transmembrane region. Most KIT mutations are located in exon 11, which codes for the juxtamembrane domain, and in exon 13 , which codes for a kinase domain.

Although amplifications or activating mutations of KIT are generally rare in melanoma, they are more commonly found in mucosal, acral and in melanomas arising from chronically sun damaged-skin. ${ }^{54}$ As the number of 
advanced melanoma patients harboring KIT mutations is low, the clinical experience of KIT inhibitors is limited.

The most widely investigated KIT inhibitor is imatinib, which is FDA-approved for gastrointestinal stromal tumors (GIST) and dermatofibrosarcoma protuberans.

Out of 51 KIT mutant melanoma patients in a phase II clinical trial, 28 patients were treated with imatinib $400 \mathrm{mg}$ orally bid. The overall response rate (ORR) was $16 \%$ with a median OS of 46.3 weeks. ${ }^{55}$ In a phase II clinical trial of 43 KIT mutant melanoma patients, 23 had an ORR (10 patients achieved PR and 13 SD). ${ }^{56} 1$-year OS was $51 \%, 6$-month PFS rate was $36.6 \%$. The best predictor of treatment response in both trials was the presence of mutation in c-KIT exons 11 and 13. Most frequently observed adverse events were hematologic toxicities (leukopenia and anemia), fatigue, nausea, rash and periorbital edema.

Nilotinib is a BCR-ABL1 TKI that was rationally designed to have increased potency and selectivity for the oncogene BCR-ABL1. It also inhibits c-KIT with greater potency than imatinib and is effective against several known $c$-KIT mutations in vitro. ${ }^{57,58}$ Nilotinib achieved a satisfactory disease control rate (DCR) in a phase II clinical study of twenty-seven melanoma patients that had progressed on imatinib or in patients with brain metastases (4-month DCR $27 \%$ and $12.5 \%$, respectively). ${ }^{59}$

The effect of nilotinib (400 $\mathrm{mg}$ bid) was investigated in another open-label single-arm clinical trial (TEAM Trial, submitted for publication). ${ }^{60}$ Similarly, among 42 patients in the nilotinib arm, the ORR was $26.2 \%$ (95\% CI, 13.9\%-42.0\%; PRs, $\mathrm{n}=11$; complete response (CR), $\mathrm{n}=0)$. The median PFS was 4.2 months $(95 \% \mathrm{CI}$, 2.1-5.8 months). At 6 months, the estimated PFS rate was $34.6 \%$ (95\% CI, $20.2 \%-49.3 \%$ ).

\section{Monoclonal antibodies}

\section{Bevacizumab}

The prognostic implications of overexpression of VEGF in clinical outcome and disease progression in melanoma remain controversial. ${ }^{61}$ VEGF is assumed to be the dominant growth factor in angiogenesis. ${ }^{62}$ The relevance of angiogenesis/neoangiogenesis in tumor metabolism, proliferation and the tumor microenvironment is unquestioned, ${ }^{63}$ thus the inhibition of the VEGF pathway offers a promising therapeutic approach.

Bevacizumab, a monoclonal antibody against VEGF-A, was the first anti-angiogenic agent on the market. It has been approved for breast neoplasms, non-small cell lung cancer, renal, ovarian, cervix and colorectal cancer. ${ }^{64} \mathrm{Ac}-$ cording to a recently published phase II clinical study, nab-paclitaxel in combination with bevacizumab showed an ORR of $36 \%$ in unresectable stage III and IV melanoma patients $(\mathrm{n}=50)$ in a first line setting. ${ }^{65}$ The following multicenter phase II clinical trial combining temozolomide (TMZ) with bevacizumab showed limited efficacy. ${ }^{66}$ On the other hand, current phase I/II clinical trials of bevacizumab in combination with ipilimumab, erlotinib or imatinib demonstrated no synergistic effect. ${ }^{67-69}$ Nevertheless, well-controlled phase III clinical trials are warranted to further investigate these results.

Tendency in clinical research opts in favor of combination therapies, since a certain immunomodulatory effect of TKIs seems to be existent. ${ }^{70,71}$ Summarizing the literature, multi-targeted TKI are not established as standard treatment of advanced melanoma but are still discussed as potential second line options in BRAF- and NRAS wt melanoma patients. ${ }^{93}$

\section{Etaracizumab}

Etaracizumab (MEDI-522), a monoclonal antibody against Integrin alphavbeta3, resulted in similar OS and PFS rates when compared to combination with DTIC (12.6 versus 9.4 months, respectively $)^{72}$ and was not further investigated in a phase III setting.

\section{Intetumumab}

The anti- $\alpha_{V}$-integrin monoclonal antibody showed only a nonsignificant trend towards an improved OS in a randomized phase II trial compared to DTIC. ${ }^{73}$

\section{mTOR-inhibitors}

Since the mammalian target of rapamycin (mTOR) signaling is upregulated in metastatic melanoma, drugs targeting mTOR seem to represent promising therapeutic targets. Everolimus (RAD-001), an orally administered inhibitor of mTOR, achieved only SD as best ORR in a cohort of 20 metastatic melanoma patients with a PFS of 3 months. ${ }^{74}$ It failed to show significant objective responses in combination with TMZ over TMZ alone. ${ }^{75}$ A phase II clinical study of Everolimus in combination with pasireotide didn't meet its primary endpoint in uveal metastatic melanoma. ${ }^{76}$

\section{Kinase inhibitors}

\section{Early developments}

The successful development of kinase inhibitors marked a milestone in the treatment of metastatic melanoma. Until 2010, no systemic treatment had demonstrated any improvement of overall survival in metastatic melanoma. Tsai et al. first discovered a potent, selective inhibitor of BRAF V600E, using a scaffold-based drug design approach. ${ }^{77}$ In preclinical studies, this compound showed impressive antitumor activity in BRAF V600E mutated cell lines by inducing cell cycle arrest and apoptosis, with no such effect on BRAF wt cell lines. Oral administration in xenograft models harboring the V600E mutation resulted 
in a substantial block of tumor growth and clinical regression without any apparent toxicity. ${ }^{77,78}$ Early clinical trials confirmed that BRAF inhibition did in fact cause complete or partial tumor regression in a large portion of patients harboring the BRAF V600 mutation. ${ }^{79,80}$

\section{BRAF inhibition}

BRAF inhibition rapidly became standard of care in $B R A F$ mutated melanoma patients. Vemurafenib is an orally bioavailable, ATP-competitive, small-molecule (489.92 Da) inhibitor of BRAF. Dabrafenib (519.56 Da) and encorafenib (540.01 Da) are similar molecules. The change in paradigm of treatment of advanced melanoma is mainly based on the results of the following two pivotal trials.

The international, multicenter phase III randomized clinical trial comparing vemurafenib to the reference chemotherapy dacarbazine (BRIM-3) showed a significantly longer mOS in the vemurafenib group (13.6 months [95\% CI 12.0-15.2] vs 9.7 months [7.9-12.8]; hazard ratio [HR] 0.70 [95\% CI 0.57-0.87]; $\mathrm{p}=0.0008$ ), as well as a significantly increased mPFS $(6.9$ months $[95 \%$ CI $6.1-7.0]$ vs 1.6 months [1.6-2.1]; HR 0.38 [95\% CI $0.32-0.46] ; p<0.0001)$. OS and PFS were significantly shorter in patients with increased LDH levels at baseline in both groups. The RR amounted to $57 \%$ in the vemurafenib group vs. $9 \%$ in the dacarbazine group. ${ }^{81,82}$ Following this phase III trial, vemurafenib was approved by the FDA in 2011 for treatment of Stage IIIC and IV metastatic melanoma patients harboring a BRAF V600E mutation.

Moreover, the multicenter phase III randomized clinical trial evaluating the BRAFi dabrafenib vs. dacarbazine (BREAK-3) showed comparable results, with a mPFS of 6.9 months for dabrafenib vs. 2.7 months for dacarbazine, hazard ratio (HR) 0.37 (95\% CI $0.23-0.57$; p $<0.0001$ ). The mOS in this study at last update was at 18.2 months vs. 15.6 months, HR 0.76 (95\% CI $0.48-1.21)$. The ORR was $50 \%$ in the dabrafenib group and $6 \%$ in the dacarbazine group. ${ }^{83,84}$ Dabrafenib received FDA approval in 2013.

Common AEs of BRAFi monotherapy with vemurafenib include arthralgia (56\% of patients), rash (41\%), fatigue (46\%) and UVA-dependent photosensitivity $(41 \%) .{ }^{85}$ The most frequent grade 3-4 side effects include cutaneous squamous cell carcinoma (19\%) and keratoacanthoma $(10 \%)$, rash (9\%) as well as abnormal liver function tests (11\%). Interestingly, $2.4 \%$ of patients developed a new primary melanoma during treatment. ${ }^{86}$ Treatment discontinuation due to SAEs occurred in $7 \%$ of patients on vemurafenib. $^{81,82}$ Common side effects of dabrafenib monotherapy comprise cutaneous AEs (hyperkeratosis $36 \%$, alopecia $27 \%$, skin papilloma $22 \%$, palmar-plantar hyperkeratosis $19 \%$, rash $30 \%$ ), pyrexia $(16 \%)$, fatigue $(18 \%)$, headache $(18 \%)$ and arthralgia (19\%). $10 \%$ of patients developed cutaneous squamous cell carcinoma or keratoacanthoma. Treatment discontinuation due to SAEs occurred in $3 \%$ of dabrafenib patients. ${ }^{83,84}$ The most common grade 3-4 AEs for dabrafenib include cutaneous squamous cell carcinoma (7\%) and pyrexia (3\%). Direct comparison of AEs in these phase III trials is difficult. In general, dabrafenib monotherapy exhibited a much lower rate of photosensitivity (2\%) compared to vemurafenib (41\%), as it has UVA-absorbing properties, while other BRAF inhibitors do not. ${ }^{85}$ Further, dabrafenib showed a lower rate of cutaneous malignancies, while exhibiting a higher frequency of pyrexia.

In summary, BRAF inhibition proved to be very efficacious in $B R A F$-mutated patients, with a high response rate and a rapid onset of response, but BRAFi monotherapy is almost invariably followed by relapse due to acquired drug resistance, most likely as a result of reactivation of MEK and ERK. ${ }^{87-89}$

\section{MEK inhibition}

After observing that $B R A F$ mutation is associated with an increased and selective sensitivity to MEK inhibition compared to $B R A F-w t$ cells, Solit et al. suggested that $B R A F$ mutant tumors were dependent on MEK activity and thus proposed MEK inhibition as a possible treatment for metastatic melanoma. ${ }^{90}$ Initial trials with MEK inhibitors (MEKi) confirmed this observation. ${ }^{91}$ MEKi are orally bioavailable, non-ATP competitive, allosteric binding inhibitors of MEK. While trametinib (615.39 Da) and binimetinib (441.23 Da) inhibit MEK 1 and 2, cobimetinib (531.31 Da) inhibits MEK1 only.

After positive phase I and II trials, monotherapy with trametinib in $B R A F$ mutant melanoma was investigated in a phase III multicenter open-label trial. Chemotherapy with dacarbazine or paclitaxel served as a comparison. The mPFS was significantly prolonged compared to chemotherapy (4.8 months vs. 1.5 months, HR 0.45 [95\% CI 0.33 to $0.63 ; \mathrm{p}<0.001]$ ). The rate of OS at 6 months improved as well, with $81 \%$ in the trametinib group and $67 \%$ in the chemotherapy group despite crossover (HR 0.54 ) [95\% CI 0.32 to $0.92 ; \mathrm{p}=0.01]^{.92}$

In a phase II trial, MEKi monotherapy with binimetinib was evaluated in patients with NRAS as well as in patients with $B R A F$ mutation, with a similar RR of $20 \%$ in $B R A F$ and NRAS-mutated patients. Thus, MEK inhibition was the first targeted therapy to show activity in patients harboring an NRAS mutation. The median PFS was 3.7 months in patients with NRAS-mutated melanoma [95\% CI 2.5-5.4] and 3.6 months [95\% CI 2.0-3.8] in patients with BRAFmutated melanoma. The difference in PFS between this and the aforementioned study might be explained by the fact that patients previously treated with ipilimumab or BRAF inhibitors were included in this trial, while being excluded from the aforementioned one. Of note, this study also showed evidence of MEKi activity in brain metastases. ${ }^{93}$ An ongoing phase III clinical trial of binimetinib in patients with advanced NRAS-mutant melanoma (NEMO) 
recently met its primary endpoint of improving progression-free survival compared to dacarbazine, with a median PFS of 2.8 months for binimetinib versus 1.5 months for dacarbazine; HR 0.62, [95\% CI 0.47-0.80], $\mathrm{p}<0.001 .{ }^{94}$

While MEK inhibition did show improvement in both PFS and OS, the RRs seem to be inferior to those shown for BRAF inhibitors. The molecular basis for this phenomenon remains unclear.

The side effect profile of MEKi differs greatly from BRAFi. The most common AEs with trametinib were rash $(57 \%)$, diarrhea (43\%) and peripheral edema (26\%). Frequent grade 3-4 AEs include hypertension (12\%), rash $(8 \%)$ and fatigue (4\%). Further side effects were fatigue, acneiform dermatitis, nausea, alopecia, hypertension, constipation and vomiting. Moreover, asymptomatic and reversible reduction in left ventricular ejection fraction (LVEF) and ocular toxic effects (blurred vision, reversible chorioretinopathy) were observed. ${ }^{95}$ Dose interruptions due to AEs occurred in $35 \%$ of patients. However, MEK inhibition did not seem to cause any cutaneous squamous-cell carcinomas or hyperproliferative skin lesions. ${ }^{92}$ While both classes have been known to cause a rash, MEKi seem to cause a papulopustular rash, while BRAFi cause a hyperkeratotic maculopapular rash. ${ }^{96}$

In addition, MEK inhibition was evaluated in combination with chemotherapy. ${ }^{97}$ However, there was no evidence for an increase in efficacy compared to MEKi monotherapy; hence, this was not evaluated in any further trials.

\section{Combined BRAF- and MEK inhibition}

With BRAF and MEK inhibitors both showing efficacy in melanoma, preclinical studies suggested an enhanced antitumor effect and a reduction of BRAFi-induced cutaneous $\mathrm{SCC}$ when combining the two classes. Due to emerging resistance to BRAFi as a result of MEK-ERK signaling reactivation, patients inevitably experience relapse. ${ }^{87,88}$ The effect of MEKi treatment after treatment resistance to BRAFi in patients harboring a $B R A F$ mutation was evaluated in a small cohort of patients, with no significant response. ${ }^{93,98}$

In contrast, early trials evaluating the combination of BRAFi and MEKi were very promising (Table 1). A randomized phase I/II clinical trial comparing the combination of dabrafenib and trametinib vs. dabrafenib alone (COMBId) showed a marked increase in PFS in the combination group (9.4 months vs. 5.8 months, HR 0.39 [95\% CI $0.25-0.62 ; \mathrm{p}<0.001])$ and a higher RR (76\% complete or partial response vs. 54\% in the monotherapy group $(\mathrm{p}=0.03))^{.99}$

Subsequently, phase III of the abovementioned trial (COMBI-d) confirmed the superiority of BRAF and MEKi combination, with a significantly prolonged mPFS of 11.0 months (95\% CI 8.0-13.9) in the dabrafenib and trametinib group vs. 8.8 months (5.9-9.3) in the dabrafenib monotherapy group (HR $0.67,95 \%$ CI $0.53-0.84 ; p=0.0004$ ). The ORR were at $69 \%$ for combination vs $53 \%$ for monotherapy $(\mathrm{p}=0.0014)$, while the mOS was 25.1 months $(95 \%$ CI 19.2-not reached) for the combination and 18.7 months (15.2-23.7) for monotherapy (hazard ratio [HR] 0.71, 95\% CI $0.55-0.92 ; \mathrm{p}=0.0107) .{ }^{100} \mathrm{~A}$ recent survival update showed landmark OS rates of $52 \%$ at 2 years and $44 \%$ at 3 years. ${ }^{101}$ The best outcome was seen in patients with normal LDH levels and less than three disease sites.

Another open-label, phase III trial comparing the combination of dabrafenib and trametinib vs. vemurafenib monotherapy (COMBI-v) was able to produce comparable results. The mPFS amounted to 11.4 months in the combination group and 7.3 months in the vemurafenib group (HR $0.56 ; 95 \%$ CI, 0.46 to $0.69 ; \mathrm{p}<0.001)$. The ORR was $64 \%$ in the combination and $51 \%$ in the vemurafenib group $(\mathrm{p}<0.001) .{ }^{102}$ Most important, quality of life was rated significantly better in the combination group using three standardized questionnaires measuring health-related quality of life during treatment and at disease progression. ${ }^{103}$

Finally, a phase III clinical trial investigating the combination of vemurafenib and cobimetinib vs. vemurafenib and placebo (coBRIM) found a significant difference in mPFS (9.9 months in the combination group vs. 6.2 months in the control group (HR 0.51; [95\% CI 0.39 to 0.68; $\mathrm{p}<0.001])$ ) and a significantly higher ORR of $68 \%$ in the combination group vs. $45 \%$ in the BRAF monotherapy group $(\mathrm{p}<0.001) .{ }^{104}$ The vemurafenib and cobimetinib combination reached a mOS of 22.3 months [95\% CI: 20.3-not reached], compared to 17.4 months for vemurafenib alone [95\% CI: 15.0-19.8], HR: 0.70; 95\% CI: $0.55-0.90, \mathrm{p}=0.005$. The OS benefit was seen in all groups, including patients with high LDH at baseline. ${ }^{105}$

Table 1

Outcome of combined BRAF and MEK inhibition of several landmark studies.

\begin{tabular}{|c|c|c|c|c|c|c|c|c|}
\hline Study & Phase & Experimental (combination) arm & mPFS (months) & mOS (months) & $1-\mathrm{yr}$ OS (\%) & 2-yr OS (\%) & 3-yr OS (\%) & Ref. \\
\hline BRIM7 & I & Vemurafenib, Cobimetinib & 13.8 & 31.2 & 83 & 64 & 37 & 106 \\
\hline coBRIM & III & Vemurafenib, Cobimetinib & 9.9 & 22.3 & $\mathrm{n} / \mathrm{a}$ & $\mathrm{n} / \mathrm{a}$ & $\mathrm{n} / \mathrm{a}$ & 105 \\
\hline Combi D & II & Dabrafenib, Trametinib & 9.4 & $\mathrm{n} / \mathrm{a}$ & 72 & 60 & 47 & 99,107 \\
\hline Combi D & III & Dabrafenib, Trametinib & 11.0 & 25.1 & 74 & 51 & 44 & 100,101 \\
\hline Combi V & III & Dabrafenib, Trametinib & 11.4 & 26.1 & 72 & 53 & 45 & 102,134 \\
\hline COLUMBUS & III & Encorafenib, Binimetinib & 14.9 & $\mathrm{n} / \mathrm{a}$ & $\mathrm{n} / \mathrm{a}$ & $\mathrm{n} / \mathrm{a}$ & $\mathrm{n} / \mathrm{a}$ & 111 \\
\hline
\end{tabular}


These data are supported by recent extended follow-up results of a phase $\mathrm{Ib}$ trial of vemurafenib and cobimetinib (BRIM7), with landmark survival for BRAF naïve patients of $82 \%, 64 \%$, and $37 \%$, respectively at 1,2 , and 3 years, and a mOS of 31.2 months. ${ }^{106}$

Addressing the question of long-term benefit, a recent update on the randomized, phase II COMBI-d trial demonstrated that there is, in fact, a subset of BRAFi-naive patients who experience long-term responses without progression on combination treatment. Normal baseline LDH levels were associated with a continued long term response. Prolonged survival was associated with normal baseline LDH levels and with fewer than 3 affected organ sites. The OS at 1,2, and 3 years for BRAFi-naive patients receiving dabrafenib at standard dose $150 \mathrm{mg}$ bid and trametinib $2 \mathrm{mg}$ qd was $72 \%, 60 \%$, and $47 \%$, respectively. In the population with normal baseline LDH levels, OS at 1,2 , and 3 years was $88 \%, 75 \%$, and $62 \%$, respectively, with a HR of $0.25(0.12-0.53) .{ }^{107}$ Thus, long term OS rates for BRAF/MEKi combination are similar to OS rates for first line anti-PD1 treatment. ${ }^{108}$ The OS may have been impacted by subsequent immunotherapy after progression on kinase inhibitors. ${ }^{107}$

Further approaches to BRAF/MEKi combination include trials with encorafenib and binimetinib. Encorafenib is a BRAFi with increased affinity to BRAF and thus a longer binding time. Results from a phase I/II clinical trial confirm a RR and PFS consistent with other BRAF/MEKi combinations (mPFS 11.3 months [95\% CI 7.4-14.6]), with a considerably higher PFS in the baseline low LDH group, going along with previous reports. ${ }^{109}$ A phase II trial evaluating encorafenib and binimetinib alone and in combination with a third agent after progression (LOGIC-2), reported an ORR of $68 \%$ for BRAF- and MEK-inhibitor naïve patients, and $20 \%$ for non-naïve patients, which is in accordance to results from other combinations. ${ }^{110}$ Recent results from a phase III trial demonstrated a mPFS of 14.9 months vs. 7.3 months for vemurafenib monotherapy with a HR of 0.54 [ $95 \%$ CI $0.41-0.71, p<0.001]$ vs. 9.6 months for encorafenib monotherapy with a HR of 0.75 [95\% CI $0.56-1.00, \mathrm{p}=0.051] .^{11}$

In general, similar percentages of grade 3-4 adverse events were seen in both monotherapy and combination treatment. Dabrafenib and trametinib combination treatment was associated with a higher frequency of pyrexia than BRAFi monotherapy (up to $71 \%$ of patients). Furthermore, gastrointestinal toxic effects (diarrhea, nausea, vomiting) were seen more frequently with combination. ${ }^{99,100,102}$ Acneiform dermatitis, a common dose-limiting effect of trametinib, was reduced in combination treatment. ${ }^{99}$ Toxic events related to paradoxical MAPK pathway activation like cutaneous SCCs and hyperkeratosis were significantly lower in all combination treatments. ${ }^{99,100,102,104,112}$

The vemurafenib/cobimetinib combination is associated with a higher frequency of central serous retinopathy, gastrointestinal events (diarrhea, nausea, vomiting), photosensitivity (due to the UVA-absorbing property of the molecule), ${ }^{85}$ elevated aminotransferase levels, and an increased creatine kinase level, with the majority of events being grade 1-2. Keratoacanthomas, cutaneous SCC, alopecia and arthralgias were observed in a lower frequency with the combination. The frequency of clinically significant cardiac events (QT-interval prolongation and decreased ejection fraction) was low and similar in monoand combination therapy, as was pyrexia. ${ }^{104}$ Encorafenib and binimetinib exhibited lower rates of pyrexia and photosensitivity than other combinations. ${ }^{109}$

Class effects of MEK blockade include reversible, asymptomatic elevated CK levels, observed in 30\% of patients in cobimetinib/vemurafenib, ${ }^{104}$ as well as transient drug-induced retinopathy, which is reversible, and can be managed with dose reduction or withdrawal of MEKi.

Overall, combination of BRAF and MEK inhibition is well tolerated and markedly delays the onset of resistance compared to BRAF monotherapy. Combination treatment consistently exhibited a lower rate of secondary cutaneous cancers compared to single drug BRAF inhibition. ${ }^{99,100,102,104}$ Long term follow-up confirms the safety, response and tolerability and suggests long-term benefit without progression for a subset of patients (approx. 20\%). ${ }^{107}$ Thus, combination of a BRAF- and MEKi is considered the current standard of care for patients harboring BRAF mutations.

\section{Perspective}

As mentioned above, the combination of a selective BRAF- and MEKi is the current standard of care. However, about $80 \%$ of patients eventually develop resistance, most notably patients with high LDH at baseline. ${ }^{107}$ Prolonged responses may be achieved by adding additional molecules (triple therapy). The choice of the third molecule could potentially be determined by individual genetic alterations. ${ }^{7}$ Possible candidates include inhibitors of cell cycle control, the PI3K-AKT pathway, and the surface receptors MET and fibroblast growth factor receptor (FGFR).

Cyclin-dependent kinases (CDKs) are serine/threonine kinases regulating cell division by promoting transitions through the cell cycle (CDK 4, 6, 2 and 1) and modulating transcription in response to several intra- and extracellular signals. ${ }^{13}$ A number of alterations concerning the p16 ${ }^{\mathrm{INK} 4 \mathrm{~A}}$ :cyclin D-CDK4/6:RB pathway have been reported in melanoma. ${ }^{7,114}$ Several different orally bioavailable, specific small molecule inhibitors of CDK 4 and 6 are currently available. By targeting CDK4/6, they inhibit phosphorylation of retinoblastoma protein and thus prevent CDK-mediated G1-S phase transition. Thus, the cell cycle is arrested in the G1 phase, thereby suppressing DNA synthesis and inhibiting growth of cancer cells.

PI3Ks are intracellular signaling proteins important for inhibition of apoptosis. The PI3K-AKT pathway was found to be activated in human cancers through multiple 
mechanisms, including activating $P I 3 K$ mutations, decreased expression or function of $P I 3 K$ suppressors (e.g. $P T E N), P I 3 K$ amplifications and activation of upstream oncogenes (e.g. NRAS) or receptors. ${ }^{7,115,116}$ Thus, multiple classes of inhibitors are available, including PI3K inhibitors (pan-isoform and isoform-specific), dual PI3K-mTOR inhibitors, AKT inhibitors and mTOR (mTORC1 and dual mTORC1/2) inhibitors. Several molecules are available for each class, many of which are currently evaluated in clinical trials. ${ }^{116}$ Initial trials combining Pan-PI3K-inhibitors with BRAF- and MEK-inhibitors have shown exceptionally high toxicity.

Most melanoma patients harboring a BRAF mutation seem to have some degree of innate resistance to kinase inhibitors. One major cause of innate resistance is stromamediated resistance. Secretion of hepatocyte growth factor (HGF) by stromal cells leads to activation of the HGFreceptor MET, reactivation of the MAPK and the PI3KAKT signaling pathways and immediate resistance to RAF inhibition. ${ }^{117}$ Consequently, dual inhibition of RAF and either MET or HGF serves as a potential strategy to counteract this mechanism of resistance. Several small molecule, highly specific inhibitors of MET and HGF are currently being tested.

Human fibroblast growth factors (FGF) are polypeptide growth factors that transduce signals by binding to transmembrane receptor tyrosine kinases, the FGFR, ${ }^{118,119}$ which then activate important cellular pathways including the MAPK and PI3K pathway. ${ }^{120}$ Amongst others, FGFs control cell proliferation, migration, angiogenesis, apoptosis and differentiation, and further play a role in neoangiogenesis, thus aiding tumor vascularization. ${ }^{121}$ Hyperactivation of FGFR signaling seems to be associated with growth and progression in several different types of cancers. ${ }^{122}$ FGF2 is overexpressed on melanoma cells, but not on normal melanocytes, ${ }^{123}$ and has been linked to tumor progression in multiple malignancies, including melanoma. ${ }^{122}$ BGJ398 is an orally bioavailable, potent and selective inhibitor of FGFRs that selectively suppresses FGFR signaling and proliferation in tumor cells with FGFR dependency and has an effect on endothelial cells by blocking FGF-induced angiogenesis, hence inhibiting tumor growth. ${ }^{124,125}$ It is currently being tested in clinical trials.

Two ongoing trials are investigating the addition of a third molecule to encorafenib and binimetinib. A phase $\mathrm{Ib} / \mathrm{II}$ trial is comparing the efficacy and safety of the triple therapy with encorafenib, binimetinib and ribociclib (a CDK4/6 inhibitor) versus the dual combination of encorafenib and binimetinib in $B R A F$-mutant metastatic melanoma (NCT01543698). A phase II clinical trial (LOGIC 2, NCT02159066) adds a third molecule based on an individual profile of molecular alterations once patients progress on encorafenib and binimetinib.

Moreover, the combination of MAPK inhibitors with immunotherapy represents an interesting therapy approach. ${ }^{126}$ Upregulation of melanocyte differentiation antigen expression by $B R A F$-mutant melanoma cells upon exposure to BRAF inhibitors has been described in several studies in human melanoma cell lines and melanoma biopsies. ${ }^{127-129}$ Similarly, MEKi also seem to increase expression of melanocyte differentiation antigens, ${ }^{127,130}$ improving antigen-specific T-cell recognition. ${ }^{127,131}$ This might result in increased lymphocyte homing to tumor cells, especially CD8+ cells, and improved lymphocyte function. ${ }^{126,128}$ Thus, efficacy of immunotherapy may be augmented by combination with kinase inhibitors. However, long-term effects are uncertain and initial clinical trials showed increased toxicity: a phase I study combining vemurafenib and ipilimumab was stopped due to liver toxicity, ${ }^{132}$ while a phase I dose-finding trial investigating the safety of the combination of dabrafenib and ipilimumab and triple therapy with dabrafenib, trametinib, and ipilimumab had to close the triple arm as intestinal perforation (following colitis) was seen in 2 out of 7 total patients. ${ }^{133}$

Targeted- and immunotherapy have both demonstrated impressive efficacy with profound impact on survival. To date, there are no convincing clinical data available that would justify one of the two as an established first line. Since this question is highly relevant for daily clinical care, careful investigation in further clinical trials is needed.

Targeted therapy with a BRAF- and MEKi combination is a reasonable first- and second line treatment option in BRAFmutated melanoma. Furthermore, MEK inhibition appears promising in NRAS mutated patients, especially after failure of immunotherapy. New combination trials are ongoing. There is justified optimism that these combinations will further improve the outcome in $B R A F$ - and $N R A S$-mutated melanoma, but possibly also in other populations such as $N F 1-$, GNA- and GNAQ-mutated melanoma. Additional benefit might be seen in the adjuvant and neoadjuvant setting.

\section{Conflict of interest statement}

RD receives research funding from Novartis, Merck Sharp \& Dohme (MSD), Bristol-Myers Squibb (BMS), Roche, GlaxoSmithKline (GSK) and has a consultant or advisory board relationship with Novartis, Merck Sharp \& Dohme, Bristol-Myers Squibb, Roche, GlaxoSmithKline and Amgen, all of which are not relevant to this manuscript. SMG serves as an intermittent consultant for BMS, MSD, Roche and Novartis; and has received travel grant support from BMS, MSD, Roche and Novartis. VCA has received honoraria from Merck Sharp \& Dohme (MSD), and funding from the Euronco Foundation (Zurich, Switzerland) and the Louis Widmer AG (Schlieren, Switzerland). No other disclosures are reported.

\section{References}

1. Vennepureddy A, Thumallapally N, Motilal Nehru V, Atallah JP, Terjanian T. Novel drugs and combination therapies for the treatment of metastatic melanoma. J Clin Med Res 2016;8(2):63-75. 
2. Kee D, McArthur G. Targeted therapies for cutaneous melanoma. Hematol Oncol Clin North Am 2014;28(3):491-505.

3. Lawrence MS, Stojanov P, Polak P, et al. Mutational heterogeneity in cancer and the search for new cancer-associated genes. Nature 2013; 499(7457):214-8.

4. Berger MF, Hodis E, Heffernan TP, et al. Melanoma genome sequencing reveals frequent PREX2 mutations. Nature 2012;485(7399):502-6.

5. Sullivan R, LoRusso P, Boerner S, Dummer R. Achievements and challenges of molecular targeted therapy in melanoma. Am Soc Clin Oncol Educ Book 2015;177-86.

6. Paluncic J, Kovacevic Z, Jansson PJ, et al. Roads to melanoma: key pathways and emerging players in melanoma progression and oncogenic signaling. Biochim Biophys Acta 2016;1863(4):770-84.

7. Genomic classification of cutaneous melanoma. Cell 2015;161(7): 1681-96.

8. Krauthammer M, Kong Y, Bacchiocchi A, et al. Exome sequencing identifies recurrent mutations in NF1 and RASopathy genes in sunexposed melanomas. Nat Genet 2015 Sep;47(9):996-1002. http:// dx.doi.org/10.1038/ng.3361 [Epub 2015 Jul 27].

9. Roh MR, Gupta S, Park KH, et al. Promoter methylation of PTEN is a significant prognostic factor in melanoma survival. J Invest Dermatol 2016 May;136(5):1002-11. http://dx.doi.org/10.1016/ j.jid.2016.01.024 [Epub 2016 Feb 15].

10. Sosman JA, Puzanov I. Molecular targets in melanoma from angiogenesis to apoptosis. Clin Cancer Res 2006;12(7 Pt 2):2376s-83s.

11. Hodis E, Watson IR, Kryukov GV, et al. A landscape of driver mutations in melanoma. Cell 2012;150(2):251-63.

12. Jovanovic B, Krockel D, Linden D, Nilsson B, Egyhazi S, Hansson J. Lack of cytoplasmic ERK activation is an independent adverse prognostic factor in primary cutaneous melanoma. J Invest Dermatol 2008;128(11):2696-704.

13. Mandala M, Merelli B, Massi D. Nras in melanoma: targeting the undruggable target. Crit Rev Oncol Hematol 2014;92(2):107-22.

14. Stephen AG, Esposito D, Bagni RK, McCormick F. Dragging ras back in the ring. Cancer Cell 2014;25(3):272-81.

15. Sullivan RJ. The role of mitogen-activated protein targeting in melanoma beyond BRAFV600. Curr Opin Oncol 2016;28(2):185-91.

16. Arafeh R, Qutob N, Emmanuel R, et al. Recurrent inactivating RASA2 mutations in melanoma. Nat Genet 2015;47(12):1408-10.

17. Shain AH, Yeh I, Kovalyshyn I, et al. The genetic evolution of melanoma from precursor lesions. N Engl J Med 2015;373(20):1926-36.

18. Long GV, Menzies AM, Nagrial AM, et al. Prognostic and clinicopathologic associations of oncogenic BRAF in metastatic melanoma. J Clin Oncol 2011;29(10):1239-46.

19. Jakob JA, Bassett Jr RL, Ng CS, et al. NRAS mutation status is an independent prognostic factor in metastatic melanoma. Cancer 2012;118(16):4014-23.

20. Chang DZ, Panageas KS, Osman I, Polsky D, Busam K, Chapman PB. Clinical significance of BRAF mutations in metastatic melanoma. J Transl Med 2004;2(1):46.

21. Ellerhorst JA, Greene VR, Ekmekcioglu S, et al. Clinical correlates of NRAS and BRAF mutations in primary human melanoma. Clin Cancer Res 2011;17(2):229-35.

22. Schlaak M, Bajah A, Podewski T, et al. Assessment of clinical parameters associated with mutational status in metastatic malignant melanoma: a single-centre investigation of 141 patients. Br J Dermatol 2013;168(4):708-16.

23. Carlino MS, Todd JR, Gowrishankar K, et al. Differential activity of MEK and ERK inhibitors in BRAF inhibitor resistant melanoma. Mol Oncol 2014;8(3):544-54.

24. Frauchiger AL, Mangana J, Rechsteiner M, et al. Prognostic relevance of LDH and serum S-100 levels in Stage IV melanoma with known BRAF mutation status. Br J Dermatol 2016 Apr;174(4): 823-30. http://dx.doi.org/10.1111/bjd.14347.

25. Meckbach D, Keim U, Richter S, et al. BRAF-V600 mutations have no prognostic impact in stage IV melanoma patients treated with monochemotherapy. PLoS One 2014;9(2):e89218.
26. Edlundh-Rose E, Egyhazi S, Omholt K, et al. NRAS and BRAF mutations in melanoma tumours in relation to clinical characteristics: a study based on mutation screening by pyrosequencing. Melanoma Res 2006;16(6):471-8.

27. Menzies AM, Haydu LE, Visintin L, et al. Distinguishing clinicopathologic features of patients with V600E and V600K BRAFmutant metastatic melanoma. Clin Cancer Res 2012;18(12): 3242-9.

28. Johnson DB, Lovly CM, Flavin M, et al. Impact of NRAS mutations for patients with advanced melanoma treated with immune therapies. Cancer Immunol Res 2015;3(3):288-95.

29. Joseph RW, Sullivan RJ, Harrell R, et al. Correlation of NRAS mutations with clinical response to high-dose IL-2 in patients with advanced melanoma. J Immunother 2012;35(1):66-72.

30. Mangana J, Cheng PF, Schindler K, et al. Analysis of BRAF and NRAS mutation status in advanced melanoma patients treated with anti-CTLA-4 antibodies: association with overall survival? PLoS One 2015;10(10):e139438.

31. Griewank KG, Murali R, Puig-Butille JA, et al. TERT promoter mutation status as an independent prognostic factor in cutaneous melanoma. J Natl Cancer Inst 2014;106(9).

32. Nagore E, Heidenreich B, Requena C, et al. TERT promoter mutations associate with fast-growing melanoma. Pigment Cell Melanoma Res 2016;29(2):236-8.

33. Lahtz C, Stranzenbach R, Fiedler E, Helmbold P, Dammann RH. Methylation of PTEN as a prognostic factor in malignant melanoma of the skin. J Invest Dermatol 2010;130(2):620-2.

34. Zwick E, Bange J, Ullrich A. Receptor tyrosine kinase signalling as a target for cancer intervention strategies. Endocr Relat Cancer 2001; 8(3):161-73.

35. Wilhelm SM, Carter C, Tang L, et al. BAY 43-9006 exhibits broad spectrum oral antitumor activity and targets the RAF/MEK/ERK pathway and receptor tyrosine kinases involved in tumor progression and angiogenesis. Cancer Res 2004;64(19):7099-109.

36. Eisen T, Ahmad T, Flaherty KT, et al. Sorafenib in advanced melanoma: a phase II randomised discontinuation trial analysis. $\mathrm{Br} J \mathrm{Can}-$ cer 2006;95(5):581-6.

37. Ott PA, Hamilton A, Min C, et al. A phase II trial of sorafenib in metastatic melanoma with tissue correlates. PLoS One 2010;5(12):e15588.

38. Flaherty K. VIII Perspectives in melanoma meeting Berlin 2004. (Sept 2004).

39. Flaherty KT, Lee SJ, Zhao F, et al. Phase III trial of carboplatin and paclitaxel with or without sorafenib in metastatic melanoma. $J$ Clin Oncol 2013;31(3):373-9.

40. Hauschild A, Agarwala SS, Trefzer U, et al. Results of a phase III, randomized, placebo-controlled study of sorafenib in combination with carboplatin and paclitaxel as second-line treatment in patients with unresectable stage III or stage IV melanoma. J Clin Oncol 2009;27(17):2823-30.

41. Eisen T, Marais R, Affolter A, et al. Sorafenib and dacarbazine as first-line therapy for advanced melanoma: phase I and open-label phase II studies. Br J Cancer 2011;105(3):353-9.

42. Amaravadi RK, Schuchter LM, McDermott DF, et al. Phase II trial of temozolomide and sorafenib in advanced melanoma patients with or without brain metastases. Clin Cancer Res 2009;15(24):7711-8.

43. McDermott DF, Sosman JA, Gonzalez R, et al. Double-blind randomized phase II study of the combination of sorafenib and dacarbazine in patients with advanced melanoma: a report from the 11715 Study Group. J Clin Oncol 2008;26(13):2178-85.

44. Urosevic-Maiwald M, Barysch MJ, Cheng PF, et al. Profiling reveals immunomodulatory effects of sorafenib and dacarbazine on melanoma. Oncoimmunology 2015;4(2):e988458.

45. Bhatia S, Moon J, Margolin KA, et al. Phase II trial of sorafenib in combination with carboplatin and paclitaxel in patients with metastatic uveal melanoma: SWOG S0512. PLoS One 2012;7(11):e48787.

46. NCT01377025. A Study of Sorafenib in Patients with Chemonaive Metastatic Uveal Melanoma (STREAM). 
47. Flaherty KT, Schiller J, Schuchter LM, et al. A phase I trial of the oral, multikinase inhibitor sorafenib in combination with carboplatin and paclitaxel. Clin Cancer Res 2008;14(15):4836-42.

48. European Medicines Agency: Votrient Product Information; 2015. [updated 27 Oct 2015]. Available from: http://www.ema.europa.eu.

49. Fruehauf JP, Alger B, Jakowatz JG, et al. A phase II single-arm study of pazopanib and paclitaxel as first-line treatment for patients with advanced melanoma. 2014 ASCO Annual Meeting. 2014 [Abstract Number: 9036]:2.

50. Kendra KL, Plummer R, Salgia R, et al. A multicenter phase I study of pazopanib in combination with paclitaxel in first-line treatment of patients with advanced solid tumors. Mol Cancer Ther 2015;14(2):461-9.

51. Thurneysen S, Cheng PF, Nagel HW, Kunz M, Jaberg-Bentele N, Nageli M, et al. An exploratory study investigating the metabolic activity and local cytokine profile in patients with melanoma treated with pazopanib and paclitaxel. Br J Dermatol 2016;175(5):966-78.

52. Algazi AP, Cha E, Ortiz-Urda SM, et al. The combination of axitinib followed by paclitaxel/carboplatin yields extended survival in advanced BRAF wild-type melanoma: results of a clinical/correlative prospective phase II clinical trial. Br J Cancer 2015;112(8):1326-31.

53. Hong DS, Kurzrock R, Falchook GS, et al. Phase 1b study of lenvatinib (E7080) in combination with temozolomide for treatment of advanced melanoma. Oncotarget 2015;6(40):43127-34.

54. Curtin JA, Busam K, Pinkel D, Bastian BC. Somatic activation of KIT in distinct subtypes of melanoma. J Clin Oncol 2006;24(26): 4340-6.

55. Carvajal RD, Antonescu CR, Wolchok JD, et al. KIT as a therapeutic target in metastatic melanoma. JAMA 2011;305(22):2327-34.

56. Guo J, Si L, Kong Y, et al. Phase II, open-label, single-arm trial of imatinib mesylate in patients with metastatic melanoma harboring c-Kit mutation or amplification. J Clin Oncol 2011;29(21):2904-9.

57. Weisberg E, Manley PW, Breitenstein W, et al. Characterization of AMN107, a selective inhibitor of native and mutant Bcr-Abl. Cancer Cell 2005;7(2):129-41.

58. Guo TH, Hajdu M, Agaram NP, et al. Mechanisms of sunitinib resistance in gastrointestinal stromal tumors harboring KIT(AY502-3ins) mutation: an in vitro mutagenesis screen for drug resistance. Clin Cancer Res 2009;15(22):6862-70.

59. Carvajal RD, Lawrence DP, Weber JS, et al. Phase II study of nilotinib in melanoma harboring KIT alterations following progression to prior KIT inhibition. Clin Cancer Res 2015;21(10):2289-96.

60. Jun Guo, Richard D. Carvajal, Reinhard Dummer, et al. Efficacy and safety of nilotinib in patients with c-Kit-mutated metastatic or inoperable melanoma: final results from the phase-2, Single-Arm TEAM Trial. 2016, [Submitted for publication].

61. Bayer-Garner IB, Hough Jr AJ, Smoller BR. Vascular endothelial growth factor expression in malignant melanoma: prognostic versus diagnostic usefulness. Mod Pathol 1999;12(8):770-4.

62. Ferrara N. Vascular endothelial growth factor as a target for anticancer therapy. Oncologist 2004;9(Suppl 1):2-10.

63. Ferrara N, Kerbel RS. Angiogenesis as a therapeutic target. Nature 2005;438(7070):967-74.

64. (EMA) EMA. European Medicines Agency. Summary of product characteristics: Bevacizumab.

65. Spitler LE, Boasberg P, O'Day S, et al. Phase II study of nabpaclitaxel and bevacizumab as first-line therapy for patients with unresectable stage III and IV melanoma. Am J Clin Oncol 2015;38(1): $61-7$.

66. von Moos R, Seifert B, Simcock M, et al. First-line temozolomide combined with bevacizumab in metastatic melanoma: a multicentre phase II trial (SAKK 50/07). Ann Oncol 2012;23(2):531-6.

67. Flaherty KT, Hamilton BK, Rosen MA, et al. Phase I/II trial of imatinib and bevacizumab in patients with advanced melanoma and other advanced cancers. Oncologist 2015;20(8):952-9.

68. Mudigonda TV, Wyman K, Spigel DR, et al. A phase II trial of erlotinib and bevacizumab for patients with metastatic melanoma Pigment Cell Melanoma Res 2016;29(1):101-3.
69. Hodi FS, Lawrence D, Lezcano C, et al. Bevacizumab plus ipilimumab in patients with metastatic melanoma. Cancer Immunol Res 2014;2(7):632-42.

70. Jayson GC, Kerbel R, Ellis LM, Harris AL. Antiangiogenic therapy in oncology: current status and future directions. Lancet $2016 \mathrm{Jul}$ 30; 388(10043):518-29.

71. Cesca M, Bizzaro F, Zucchetti M, Giavazzi R. Tumor delivery of chemotherapy combined with inhibitors of angiogenesis and vascular targeting agents. Front Oncol 2013;3:259.

72. Hersh EM, Akporiaye E, Harris D, et al. Phase I study of immunotherapy of malignant melanoma by direct gene transfer. Hum Gene Ther 1994;5(11):1371-84.

73. O'Day S, Pavlick A, Loquai C, et al. A randomised, phase II study of intetumumab, an anti-alphav-integrin $\mathrm{mAb}$, alone and with dacarbazine in stage IV melanoma. Br J Cancer 2011;105(3):346-52.

74. Rao RD, Windschitl HE, Allred JB, et al. Phase II trial of the mTOR inhibitor everolimus (RAD-001) in metastatic melanoma JCO. 2006; Abstract presentation from the 2006 ASCO Annual Meeting.

75. Dronca RS, Allred JB, Perez DG, et al. Phase II study of temozolomide (TMZ) and everolimus (RAD001) therapy for metastatic melanoma: a North Central Cancer Treatment Group study, N0675. Am J Clin Oncol 2014:37(4):369-76.

76. Shoushtari AN, Ong LT, Schoder H, et al. A phase 2 trial of everolimus and pasireotide long-acting release in patients with metastatic uveal melanoma. Melanoma Res 2016;26(3):272-7.

77. Tsai J, Lee JT, Wang W, et al. Discovery of a selective inhibitor of oncogenic B-Raf kinase with potent antimelanoma activity. Proc Natl Acad Sci USA 2008;105(8):3041-6.

78. Joseph EW, Pratilas CA, Poulikakos PI, et al. The RAF inhibitor PLX4032 inhibits ERK signaling and tumor cell proliferation in a V600E BRAF-selective manner. Proc Natl Acad Sci USA 2010; 107(33):14903-8.

79. Bollag G, Hirth P, Tsai J, et al. Clinical efficacy of a RAF inhibitor needs broad target blockade in BRAF-mutant melanoma. Nature 2010;467(7315):596-9.

80. Flaherty KT, Puzanov I, Kim KB, et al. Inhibition of mutated, activated BRAF in metastatic melanoma. N Engl J Med 2010;363(9): $809-19$.

81. Chapman PB, Hauschild A, Robert C, et al. Improved survival with vemurafenib in melanoma with BRAF V600E mutation. $N$ Engl $J$ Med 2011;364(26):2507-16.

82. McArthur GA, Chapman PB, Robert C, et al. Safety and efficacy of vemurafenib in $\mathrm{BRAF}(\mathrm{V} 600 \mathrm{E})$ and $\mathrm{BRAF}(\mathrm{V} 600 \mathrm{~K})$ mutation-positive melanoma (BRIM-3): extended follow-up of a phase 3, randomised, open-label study. Lancet Oncol 2014;15(3):323-32.

83. Hauschild A, Grob JJ, Demidov LV, et al. Dabrafenib in BRAFmutated metastatic melanoma: a multicentre, open-label, phase 3 randomised controlled trial. Lancet 2012:380(9839):358-65.

84. Hauschild A, Grob JJ, Demidov LV, et al. An update on BREAK-3, a phase III, randomized trial: dabrafenib (DAB) versus dacarbazine (DTIC) in patients with BRAF V600E-positive mutation metastatic melanoma (MM). ASCO Meeting Abstracts31(15_suppl):9013.

85. Dummer R, Rinderknecht J, Goldinger SM. Ultraviolet A and photosensitivity during vemurafenib therapy. N Engl J Med 2012;366(5): $480-1$.

86. Zimmer L, Hillen U, Livingstone E, et al. Atypical melanocytic proliferations and new primary melanomas in patients with advanced melanoma undergoing selective BRAF inhibition. $J$ Clin Oncol 2012;30(19):2375-83.

87. Johannessen CM, Boehm JS, Kim SY, et al. COT drives resistance to RAF inhibition through MAP kinase pathway reactivation. Nature 2010;468(7326):968-72.

88. Nazarian R, Shi H, Wang Q, et al. Melanomas acquire resistance to B-RAF(V600E) inhibition by RTK or N-RAS upregulation. Nature 2010;468(7326):973-7.

89. Dummer R, Flaherty KT. Resistance patterns with tyrosine kinase inhibitors in melanoma: new insights. Curr Opin Oncol 2012;24(2):150-4. 
90. Solit DB, Garraway LA, Pratilas CA, et al. BRAF mutation predicts sensitivity to MEK inhibition. Nature 2006;439(7074):358-62.

91. Gilmartin AG, Bleam MR, Groy A, et al. GSK1120212 (JTP-74057) is an inhibitor of MEK activity and activation with favorable pharmacokinetic properties for sustained in vivo pathway inhibition. Clin Cancer Res 2011;17(5):989-1000.

92. Flaherty KT, Robert C, Hersey P, et al. Improved survival with MEK inhibition in BRAF-mutated melanoma. N Engl J Med 2012;367(2): 107-14.

93. Ascierto PA, Schadendorf D, Berking C, et al. MEK162 for patients with advanced melanoma harbouring NRAS or Val600 BRAF mutations: a non-randomised, open-label phase 2 study. Lancet Oncol 2013;14(3):249-56.

94. Dummer R, Schadendorf D, Ascierto PA, et al. Results of NEMO: a phase III trial of binimetinib (BINI) vs dacarbazine (DTIC) in NRASmutant cutaneous melanoma. ASCO Meeting Abstracts34(15_suppl): 9500.

95. Urner-Bloch U, Urner M, Stieger P, et al. Transient MEK inhibitorassociated retinopathy in metastatic melanoma. Ann Oncol 2014; 25(7): 1437-41.

96. Schad K, Baumann Conzett K, Zipser MC, et al. Mitogen-activated protein/extracellular signal-regulated kinase kinase inhibition results in biphasic alteration of epidermal homeostasis with keratinocytic apoptosis and pigmentation disorders. Clin Cancer Res 2010;16(3): 1058-64.

97. Robert C, Dummer R, Gutzmer R, et al. Selumetinib plus dacarbazine versus placebo plus dacarbazine as first-line treatment for BRAF-mutant metastatic melanoma: a phase 2 double-blind randomised study. Lancet Oncol 2013;14(8):733-40.

98. Kirkwood JM, Bastholt L, Robert C, et al. Phase II, open-label, randomized trial of the MEK1/2 inhibitor selumetinib as monotherapy versus temozolomide in patients with advanced melanoma. Clin Cancer Res 2012;18(2):555-67.

99. Flaherty KT, Infante JR, Daud A, et al. Combined BRAF and MEK inhibition in melanoma with BRAF V600 mutations. N Engl J Med 2012;367(18):1694-703.

100. Long GV, Stroyakovskiy D, Gogas H, et al. Dabrafenib and trametinib versus dabrafenib and placebo for Val600 BRAF-mutant melanoma: a multicentre, double-blind, phase 3 randomised controlled trial. Lancet 2015;386(9992):444-51.

101. Flaherty K, Davies MA, Grob JJ, et al. Genomic analysis and 3-y efficacy and safety update of COMBI-d: a phase 3 study of dabrafenib (D) + trametinib (T) vs D monotherapy in patients (pts) with unresectable or metastatic BRAF V600E/K-mutant cutaneous melanoma. ASCO Meeting Abstracts34(15_suppl):9502.

102. Robert C, Karaszewska B, Schachter J, et al. Improved overall survival in melanoma with combined dabrafenib and trametinib. N Engl J Med 2015;372(1):30-9.

103. Grob JJ, Amonkar MM, Karaszewska B, et al. Comparison of dabrafenib and trametinib combination therapy with vemurafenib monotherapy on health-related quality of life in patients with unresectable or metastatic cutaneous BRAF Val600-mutation-positive melanoma (COMBI-v): results of a phase 3, open-label, randomised trial. Lancet Oncol 2015;16(13):1389-98.

104. Larkin J, Ascierto PA, Dreno B, et al. Combined vemurafenib and cobimetinib in BRAF-mutated melanoma. N Engl J Med 2014;371(20): 1867-76.

105. Ascierto PA, McArthur GA, Dréno B, et al. coBRIM: a phase 3, doubleblind, placebo-controlled study of vemurafenib versus vemurafenib + cobimetinib in previously untreated BRAFV600 mutation-positive patients with unresectable locally advanced or metastatic melanoma (NCT01689519). J Transl Med 2015;13(1):1-2.

106. Daud A, Pavlick AC, Ribas A, et al. Extended follow-up results of a phase 1B study (BRIM7) of cobimetinib (C) and vemurafenib (V) in BRAFmutant melanoma. ASCO Meeting Abstracts34(15_suppl):9510.

107. Long GV, Weber JS, Infante JR, et al. Overall survival and durable responses in patients with BRAF V600-Mutant metastatic melanoma receiving dabrafenib combined with trametinib. $J$ Clin Oncol 2016;34(8):871-8.

108. Robert C, Long GV, Brady B, et al. Nivolumab in previously untreated melanoma without BRAF mutation. N Engl J Med 2015; 372(4):320-30

109. Sullivan RJ, Weber JS, Patel SP, et al. A phase Ib/II study of BRAF inhibitor (BRAFi) encorafenib (ENCO) plus MEK inhibitor (MEKi) binimetinib (BINI) in cutaneous melanoma patients naive to BRAFi treatment. ASCO Meeting Abstracts33(15_suppl):9007.

110. Dummer R, Sandhu S, Hassel JC, et al. LOGIC2: phase 2, multicenter, open-label study of sequential encorafenib/binimetinib combination followed by a rational combination with targeted agents after progression, to overcome resistance in adult patients with locally advanced or metastatic BRAF V600 melanoma. Eur Cancer Congr 2015. Vienna, Austria 2015.

111. Dummer R, Acierto PA, Gogas HJ, et al., editors. Results of COLUMBUS Part 1: a Phase 3 Trial of Encorafenib (ENCO) Plus Binimetinib (BINI) versus Vemurafenib (VEM) or ENCO in BRAF-Mutant Melanoma. Society for Melanoma Research 2016; Boston, MA, US.

112. Dummer R, Tsao H, Robert C. How cutaneous eruptions help to understand the mode of action of kinase inhibitors. Br J Dermatol 2012; 167(5):965-7.

113. Malumbres M. Cyclin-dependent kinases. Genome Biol 2014;15(6): 122.

114. Lee B, Sandhu S, McArthur G. Cell cycle control as a promising target in melanoma. Curr Opin Oncol 2015;27(2):141-50.

115. Aziz SA, Jilaveanu LB, Zito $C$, et al. Vertical targeting of the phosphatidylinositol-3 kinase pathway as a strategy for treating melanoma. Clin Cancer Res 2010;16(24):6029-39.

116. Kwong LN, Davies MA. Navigating the therapeutic complexity of PI3K pathway inhibition in melanoma. Clin Cancer Res 2013; 19(19):5310-9.

117. Straussman R, Morikawa $\mathrm{T}$, Shee $\mathrm{K}$, et al. Tumour microenvironment elicits innate resistance to RAF inhibitors through HGF secretion. Nature 2012;487(7408):500-4.

118. Beenken A, Mohammadi M. The FGF family: biology, pathophysiology and therapy. Nat Rev Drug Discov 2009;8(3):235-53.

119. Powers CJ, McLeskey SW, Wellstein A. Fibroblast growth factors, their receptors and signaling. Endocr Relat Cancer 2000;7(3):165-97.

120. Acevedo VD, Ittmann M, Spencer DM. Paths of FGFR-driven tumorigenesis. Cell Cycle 2009;8(4):580-8.

121. Presta M, Dell'Era P, Mitola S, Moroni E, Ronca R, Rusnati M. Fibroblast growth factor/fibroblast growth factor receptor system in angiogenesis. Cytokine Growth Factor Rev 2005;16(2):159-78.

122. Jeffers M, LaRochelle WJ, Lichenstein HS. Fibroblast growth factors in cancer: therapeutic possibilities. Expert Opin Ther Targets 2002; 6(4):469-82.

123. Metzner T, Bedeir A, Held G, et al. Fibroblast growth factor receptors as therapeutic targets in human melanoma: synergism with BRAF inhibition. J Invest Dermatol 2011;131(10):2087-95.

124. Goke A, Goke R, Ofner A, Herbst A, Lankat-Buttgereit B. The FGFR inhibitor NVP-BGJ398 induces NSCLC cell death by activating caspase-dependent pathways as well as caspase-independent apoptosis. Anticancer Res 2015;35(11):5873-9.

125. Guagnano V, Furet P, Spanka C, et al. Discovery of 3-(2,6-dichloro3,5-dimethoxy-phenyl)-1-\{6-[4-(4-ethyl-piperazin-1-yl)-phenylamin o]-pyrimidin-4-yl\}-1-methyl-urea (NVP-BGJ398), a potent and selective inhibitor of the fibroblast growth factor receptor family of receptor tyrosine kinase. J Med Chem 2011;54(20):7066-83.

126. Hu-Lieskovan S, Robert L, Homet Moreno B, Ribas A. Combining targeted therapy with immunotherapy in BRAF-mutant melanoma: promise and challenges. J Clin Oncol 2014;32(21):2248-54.

127. Boni A, Cogdill AP, Dang P, et al. Selective BRAFV600E inhibition enhances T-cell recognition of melanoma without affecting lymphocyte function. Cancer Res 2010;70(13):5213-9.

128. Frederick DT, Piris A, Cogdill AP, et al. BRAF inhibition is associated with enhanced melanoma antigen expression and a more 
favorable tumor microenvironment in patients with metastatic melanoma. Clin Cancer Res 2013;19(5):1225-31.

129. Sapkota B, Hill CE, Pollack BP. Vemurafenib enhances MHC induction in BRAF homozygous melanoma cells. Oncoimmunology 2013; 2(1):e22890.

130. Kono M, Dunn IS, Durda PJ, et al. Role of the mitogen-activated protein kinase signaling pathway in the regulation of human melanocytic antigen expression. Mol Cancer Res 2006;4(10):779-92.

131. Donia M, Fagone P, Nicoletti F, et al. BRAF inhibition improves tumor recognition by the immune system: potential implications for combinatorial therapies against melanoma involving adoptive T-cell transfer. Oncoimmunology 2012;1(9):1476-83.
132. Ribas A, Hodi FS, Callahan M, Konto C, Wolchok J. Hepatotoxicity with combination of vemurafenib and ipilimumab. $N$ Engl J Med 2013;368(14):1365-6.

133. Minor DR, Puzanov I, Callahan MK, Hug BA, Hoos A. Severe gastrointestinal toxicity with administration of trametinib in combination with dabrafenib and ipilimumab. Pigment Cell Melanoma Res 2015;28(5):611-2.

134. Robert C, Karaszewska B, Schachter J, et al. Three-year estimate of overall survival in COMBI-v, a randomized phase 3 study evaluating first-line dabrafenib (D) + trametinib (T) in patients (pts) with unresectable or metastatic BRAF V600E/K-mutant cutaneous melanoma. Ann Oncol 2016;27(suppl 6). 